\title{
Environmental impact assessment of new district developments
}

\author{
F. Fadli ${ }^{1}$, M. Sobhey ${ }^{1}$, R. Asadi ${ }^{1}$ \& E. Elsarrag ${ }^{2}$ \\ ${ }^{1}$ Department of Architecture and Urban Planning, \\ Qatar University, Qatar \\ ${ }^{2}$ Gulf Organization for Research and Development, Qatar
}

\begin{abstract}
Over the last decade, the term "sustainability" has figured prominently in city planning discussions aiming to create comfortable places to live, work, learn and relax. In an accelerated world, the need to set standards that guide city development becomes necessary and important. In the case of Qatar, where globalization has proclaimed new types of projects and facilities, GSAS, the Global Sustainability Assessment System (formerly known as QSAS, Qatar Sustainability Assessment System) has emerged as a new local initiative that aims to sustain existing and upcoming projects. The paper aims to assess a defined urban development typology "the districts development"; and tests a GSAS district new tool in order to enhance it. While the first part of the paper introduces the theoretical framework for environmental impact assessment (EIA) and develops a comparative analysis between two main international systems; the second part focuses on selected criteria within the district development and their implementation in the GSAS district tool. Finally, a set of framework and design guidelines is developed in order to refine the GSAS districts emerging tool.

Keywords: environmental impact assessment (EIA), GSAS/QSAS, district developments, sustainability assessment, Qatar/GCC.
\end{abstract}

\section{Introduction}

Environmental Impact Assessment (EIA) is commonly defined as the process by which anticipated effects on the environment of a proposed development or project 
are measured. If the likely effects are unacceptable, design measures and related mitigation measures can be taken to reduce and/or avoid those effects (EPA [3]).

The main purpose of EIA is to facilitate the systematic consideration of environmental issues as part of developmental decision-making. It does so primarily by assembling and analyzing information on the potential environmental effects of specific development proposals and how they can be prevented or mitigated. EIA takes place before major decisions are taken and, ideally, while feasible alternatives and options to proposed actions are still open. In this context, the decision-making process extends from project initiation to implementation. Thus, there are a number of key stages at which EIA can build environmental considerations into project planning and design; therefore, it is a process rather than a one-time task. EIA influences many stages over a considerable period of time and is not aimed only at producing a report for the final approval stage (Abaza et al. [1]).

\section{Generic analysis of the EIA processes; pros and cons}

As shown in Figure 1, EIA processes encounter positive as well as negative feedback while being conducted. These can be summarized briefly in the following points:

- Improve project design/siting;

- Develop informed decision-making (involving public);

- Design environmentally sensitive decisions;

- Increase accountability and transparency during the development process;

- Improve integration of projects into their environmental and social setting;

- Reduce environmental damage;

- Develop acute contribution towards achieving sustainability;

- Abaza et al. [2].

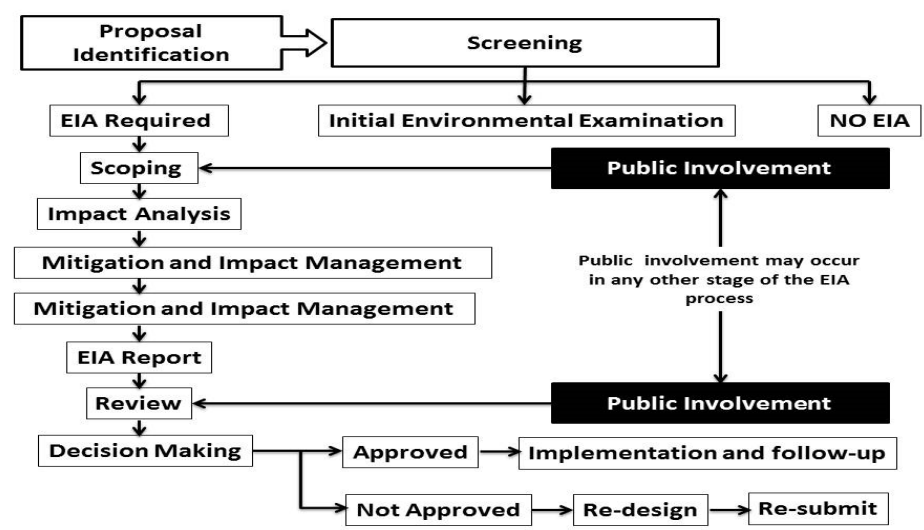

Figure 1: $\quad$ EIA process (UNEP [6]). 


\section{Global Sustainability Assessment System (G/QSAS)}

The development of GSAS/QSAS rating schemes works on a ground-up approach, to allow for seamless integration between the country's specific requirements and sustainable goals.

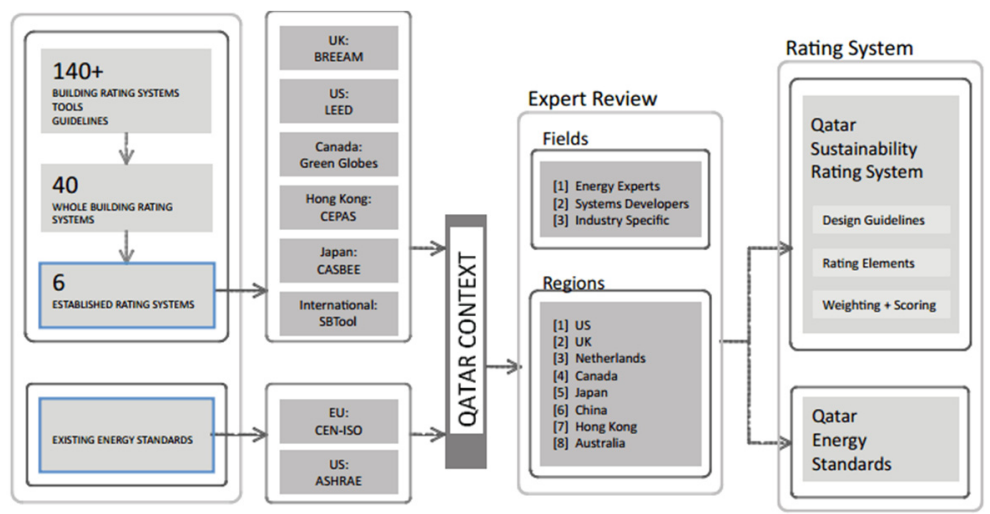

Figure 2: The development process of G/QSAS (GORD [4]).

The establishment of G/QSAS was important as to adapt to the specific characteristics of the region/country. Accordingly, it is not possible to use the same rating tool(s) in different geographic locations. G/QSAS consists of a series of sustainability indicator categories, each with a direct impact on environmental stress mitigation. The categories define these broad impacts and address ways in which a project can mitigate the negative environmental effects. The categories are sub-divided into specific criteria that measure and define specific issues. These issues range from a thorough review of water consumption to an assessment of light quality. Each criterion specifies a process for measuring individual aspects of environmental impact, the degree to which the requirements have been met. A score is then awarded to each criterion based on the degree of compliance (GORD [4]).

\section{Research aim and objectives}

The main aim of this study is to investigate issues related to the sustainability of district developments and assess their sustainability levels using an adapted version of GSAS/QSAS. To do so it is also necessary to test and enhance the new emerging tool of GSAS (urban) districts. The research main stages are explained below and clarified in Figure 3.

\subsection{A-screening: selection of the case study}

Districts have to be designed as healthy liveable places to live, work, play and learn. Hence, a set of guidelines has to be elaborated in order to provide guidance 
for the effective implementation of sustainable urban districts. In a developing country like Qatar, the need to investigate the emerging districts is very important. Data availability has directed and limited the study. Case studies are named as A and $\mathrm{B}$ for confidentiality purposes. Yet one typology is studied, the district/neighbourhood developments. The detailed description of the case study typology will be mentioned in the scoping stage.

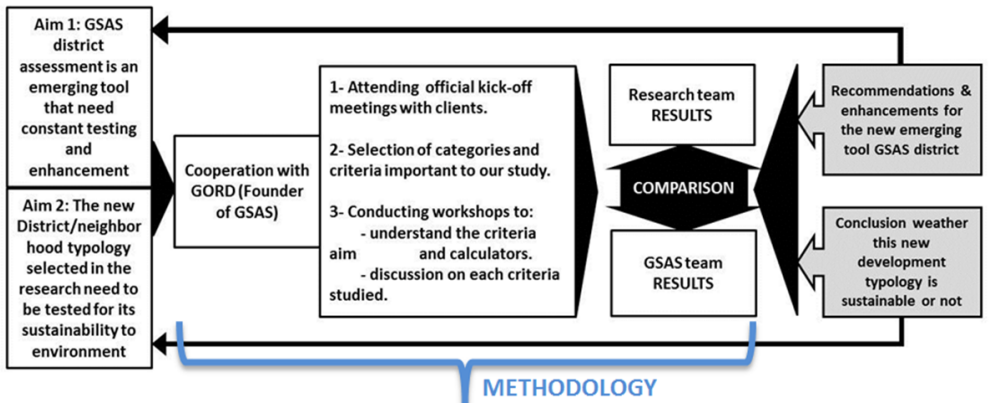

Figure 3: $\quad$ Research design and stages.

Issues to be considered in this type of district/neighbourhood development are:

- New infrastructures: New districts should be studied with relation to adjacent infrastructure, their mutual effects and demands on the available resources.

- Quality of urban life: Public spaces, walkable areas, mixed use facilities and parking footprints are issues to be considered. These can only be measured on large scale developments such as neighborhoods or districts.

- Outdoor environments: Buildings do not exist alone, they are a part of the holistic built environment where the environmental quality shall be monitored to macro and meso-scale factors such as thermal comfort, and air quality.

- Cultural and economic values: The districts' design shall enhance the cultural values of the existing community.

- Materials: Material extraction, processing and manufacturing are important stages to be looked at on a wider scale, the site scale.

- Energy consumption: On a district scale, energy consumption becomes a critical issue, where the depletion of fossil energy over the multiple services should be monitored and reduced.

- Water consumption: Water preservation is required with implementing new means of strategies for supplying and treating the several types of water.

\subsection{B-scoping: districts/neighbourhoods specs - mixed-use developments}

1- Scale of the development should be the scale of a district or a development that contains a number of districts.

2- The developments have modern architectural style buildings with high-tech design. 
3- Developments with unique and new infrastructure, for example: district cooling technique.

4- Developed on new lands on the periphery of the city, no/little adjacent developments, and served with highways.

5- Development design should comply with the characteristics of the hot arid zone climate conditions.

\section{Impact assessment and analysis}

The present detailed analysis and investigation has been carried out based on the following criteria and their calculators;

\subsection{Urban connectivity [UC] (weight 10\%)}

The Urban Connectivity category consists of factors associated with the urban environment such as zoning, transportation networks and loadings. Environmental impacts resulting from unsustainable urban practices include: climate change, fossil fuel depletion, water depletion, materials depletion, land use and contamination, water pollution, air pollution, human comfort and health (GORD [5]).

Table 1: Urban connectivity criteria.

\begin{tabular}{|c|c|c|c|}
\hline No. & Criteria & Min. score & Max. score \\
\hline UC. 1 & Transportation load & 0 & 3 \\
\hline UC. 2 & Proximity to existing districts & 0 & 3 \\
\hline UC.3 & Acoustics conditions & 0 & 3 \\
\hline UC.4 & Solid waste load & 0 & 3 \\
\hline \multicolumn{2}{|c|}{ Total possible } & 0 & 12 \\
\hline
\end{tabular}

\section{2 [UC.2] proximity to existing districts}

This criterion encourages developments near existing urban areas to maximize shared use of infrastructure.

Measurement: all projects will complete the Proximity to Existing Districts Calculator and identification on a site map developed, undeveloped, and nondevelopable land plots that are located within $1 \mathrm{~km}$ of the site boundary [5]. Score ( $\%$ of development area $\mathrm{X}$ ): $0=\mathrm{X}<45 \%, 1=45 \% \leq \mathrm{X}<60 \%, 2=60 \% \% \leq \mathrm{X}<75 \%$, $3=\mathrm{X} \geq 75 \%$.

\subsection{Site [S]: (weight 20\%)}

The site category consists of factors associated with land use, such as land conservation or remediation, site selection, planning and development. Environmental impacts resulting from improper land use and unsustainable practices include: climate change, fossil fuel depletion, water depletion, and human comfort and health (GORD [5]). 
Table 2: $\quad$ Site criteria.

\begin{tabular}{|c|c|c|c|}
\hline No. & Criteria & Min. score & Max. score \\
\hline S.1 & Land preservation & -1 & 3 \\
\hline S.2 & Water body preservation & -1 & 3 \\
\hline S.3 & Habitat preservation & -1 & 3 \\
\hline S.4 & Vegetation & -1 & 3 \\
\hline S.5 & Walkability & -1 & 3 \\
\hline S.6 & Bike-ability & -1 & 3 \\
\hline S.7 & Desertification & -1 & 3 \\
\hline S.8 & Parking footprint & -1 & 3 \\
\hline S.9 & Mixed use & -1 & 3 \\
\hline S.10 & Crime prevention & -1 & 3 \\
\hline S.11 & Public space & -1 & 3 \\
\hline S.12 & GSAS rated typologies & -1 & 3 \\
\hline & Total Possible & -12 & 36 \\
\hline
\end{tabular}

\subsection{Site: [S.5] walkability}

Supports sustainable infrastructure through development of efficient, user-friendly pedestrian pathways. All projects will complete the Walkability Calculator to determine the extent and usability of the projects pedestrian pathways.

Measurement: The project will determine the ratio of pedestrian pathway length (meters) to vehicular roadway length (meters) to evaluate the extent of pathways within the development. Additionally, the project will perform a shading simulation to compute the percent of applicable pedestrian pathways shaded by both landscape features and buildings in order to determine the usability of pathways within the development GORD [5].

Score (ratio of pedestrian pathway length to vehicular roadway length [a]):

$$
-1=\mathrm{a}<1.25,0=1.25 \leq \mathrm{a} \leq 1.5,1=1.5 \leq \mathrm{a} \leq 1.75,2=1.75 \leq \mathrm{a} \leq 2,3=\mathrm{a} \geq 2 .
$$

Score $(\%$ of pedestrian pathway shaded $[\mathrm{b}])$ :

$$
-1=b<60 \%, 0=60 \% \leq b \leq 70 \%, 1=70 \% \leq b \leq 80 \%, 2=80 \% \leq b \leq 90 \%, 3=b \geq 90 \% \text {. }
$$

\subsection{Site: [S.6] bike-ability}

Encourages sustainable infrastructure through the development of efficient, userfriendly bicycle pathways. All projects will complete the Bike-ability Calculator to determine the extent of bicycle pathways. Bicycle pathways may occur along roadways as bike lanes or as separated bike paths through public spaces. Pedestrian pathways may not be counted as bicycle pathways.

Measurements: the Bike-ability Calculator computes the criterion score based on the ratio of bicycle pathway length (meters) to total vehicular roadway length (meters). Bicycle pathways are separate from pedestrian pathways and may occur along roadways, within public spaces, or between buildings with signage [5].

Score (ratio of bicycle pathway length to vehicular roadway length $[\mathrm{X}]$ ): 
$-1=X<0.075,0=0.075 \leq X \leq 0.1,1=0.1 \leq X \leq 0.125,2=0.125 \leq X \leq 0.15,3=X \geq 0.15$.

\subsection{Site: [S.9] mixed use}

Aims to maximize the number of major uses within the development in order to reduce the need for transport. Measurement: All projects will complete the Mixed Use Calculator to determine the number of diverse uses within walking distance to the proposed population. Here, the uses are defined by the following categories: residential, office space, public services, places of worship, retail - services, retail - goods, and retail - food.

The Mixed Use Calculator computes the criterion score based on the population and number of uses within each 480 meter grid block, delineated by the project on a site plan. The number of uses is weighted by the population in order to calculate a Performance Indicator GORD [5].

Score (performance indicator $[\mathrm{X}]$ ):

$$
-1=\mathrm{X}<1,0=1 \leq \mathrm{X}<2,1=2 \leq \mathrm{X}<3,2=3 \leq \mathrm{X}<4,3=\mathrm{X} \geq 4 .
$$

\subsection{Site: $[$ S.11] public space}

Encourage social interaction and promote the physical and mental well-being of the community by providing accessible and usable outdoor public space. All projects will provide an adequate amount of public space for the district users, as well as ensuring that the public spaces are easily accessible and include appropriate levels of shading.

Measurements: all projects will complete the Public Space Calculator to determine the accessibility, area, and usability of public space. Public spaces can include parks, plazas, recreational facilities, sports fields, community facilities, and other spaces that are open and accessible to the general public. The calculator computes a Public Space Performance Indicator based on the public space per capita and the accessibility of public spaces to the development users. Additionally, the project will perform a shading simulation to determine the percent of public spaces shaded (GORD [5]).

Score (public space performance indicator $[\mathrm{a}])$ :

$$
-1=a<15,0=15 \leq a<20,1=20 \leq a<25,2=25 \leq a<30,3=a \geq 30 .
$$

Score ( $\%$ of public space shaded $[\mathrm{b}])$ :

$-1=b<25 \%, 0=25 \% \leq b<30 \%, 1=30 \% \leq b<35 \%, 2=35 \% \leq b<40 \%, 3=b \geq 40 \%$.

\subsection{Energy [E]: (Ct-weight 18\%)}

The energy category consists of factors associated with the efficiency of energy delivery and the use of fossil energy sources that result in harmful emissions and pollution. Negative impacts resulting from energy use and unsustainable practices include: climate change, fossil fuel depletion, air pollution, and human comfort and health. Factors that could mitigate environmental impacts due to energy use 
include: selecting efficient building systems, lowering the demand on nonrenewable sources of energy, thereby reducing the depletion of fossil fuels, reducing harmful emissions, and minimizing the amount of harmful substances produced by the energy delivery systems and the energy supply network (GORD [5]).

Table 3: $\quad$ Energy criteria.

\begin{tabular}{|c|c|c|c|}
\hline No. & Criteria & Min. score & Max. score \\
\hline E.2 & Energy delivery performance & -1 & 3 \\
\hline E.3 & Fossil fuel depletion & -1 & 3 \\
\hline E.4 & $\mathrm{CO}_{2}$ emission & -1 & 3 \\
\hline E.5 & $\mathrm{NO}_{2}, \mathrm{SO}_{2}$, and particulate matter & -1 & 3 \\
\hline \multicolumn{2}{|r|}{ Total possible } & -4 & 12 \\
\hline
\end{tabular}

\subsection{Energy: [E.2] energy delivery performance}

Establishes energy delivery performance of all systems that serve the district. All projects will conduct assessments of integrated district energy performance in relation to the baseline and targets outlined in the District Energy Performance Calculator.

Measurements: all projects will complete the District Energy Performance Calculator to determine the District's Delivered Energy Performance Coefficient (EPCdel). The district's energy delivery performance $\left(E_{d e l}\right)$ is based on several input parameters including, but not limited to: 3 . infrastructure delivered energy: water supply energy performance, wastewater treatment energy performance, district cooling plant pump energy performance, irrigation energy performance, park lighting energy performance, traffic lighting energy performance, street lighting energy performance (GORD [5]).

Score $\left(\mathrm{EPC}_{\mathrm{del}}\right.$ value):

$$
-1=\mathrm{EPC}>1,0=0.8<\mathrm{EPC} \leq 1,1=0.7<\mathrm{EPC} \leq 0.8,2=0.6<\mathrm{EPC} \leq 0.7,3=\mathrm{EPC} \leq 0.6 \text {. }
$$

\subsection{Outdoor environment [OE] (CT weight 7\%)}

The outdoor environment category consists of factors associated with outdoor environmental quality such as heat island effect, adverse winds, air flow, and acoustic quality within the district. Impacts resulting from ineffective control and design of the outdoor environment include climate change, fossil, fuel depletion and human comfort and health. In this it is important to:

- Maximize the vegetation and solar reflectiveness to reduce the impact of a heat island effect.

- $\quad$ Protect spaces in the district from adverse wind conditions.

- Ensure a sufficient level of air flow to allow for the potential to naturally ventilate buildings.

- $\quad$ Minimize the amount of noise produced within the development (GORD [5]). 
Table 4: Outdoor environment criteria.

\begin{tabular}{|c|c|c|c|}
\hline No. & Criteria & Min. score & Max. score \\
\hline OE.1 & Heat island effect & -1 & 3 \\
\hline OE.2 & Adverse wind conditions & -1 & 3 \\
\hline OE.3 & Air flow & -1 & 3 \\
\hline OE.4 & Acoustic quality & -1 & 3 \\
\hline \multicolumn{2}{|c|}{ Total possible } & -4 & 12 \\
\hline
\end{tabular}

\subsection{Outdoor environment [OE.1] UHI effect}

This criterion aims to minimize the heat island effect and reduce the impact on the surrounding habitat and environment. All projects are required to develop strategies and perform calculations to ensure that the heat island effect is controlled.

Measurements: through this assessment, the projects are required to specify the solar reflectance value and area for all surface areas within the site, including both ground surfaces and building rooftops for pre- and post-developments. The difference between pre- and post-development overall solar reflectance values is used as an indicator to evaluate the performance of the development in minimizing the heat island effect (GORD [5]).

Score (performance indicator $[\mathrm{X}]$ ):

$$
\begin{gathered}
-1=\mathrm{X}<-0.3,0=-0.3 \leq \mathrm{X}<-0.2,1=-0.2 \leq \mathrm{X}<-0.1, \\
2=-0.1 \leq \mathrm{X}<0,3=\mathrm{X} \geq 0 .
\end{gathered}
$$

\subsection{Environment [OE.2] adverse wind conditions}

The adverse wind condition criterion aims to minimize adverse wind conditions to surrounding spaces at the pedestrian level. All the projects here are required to develop strategies and perform wind control studies to minimize wind exposure.

Measurements: all projects will perform computational fluid dynamics (CFD) simulations, pre- and post-development, to determine whether the increase in average wind speed, at the pedestrian level, is within the predefined reference threshold. Pre- and post-development average wind speeds should be located and measured at the corners of all collector and local road intersections. The reference threshold is defined as either an increase of $2 \mathrm{~m} / \mathrm{s}$ or less from pre- to postdevelopment OR a post-development wind speed of less than or equal to $5.5 \mathrm{~m} / \mathrm{s}$ GORD [5].

Score $(\%$ of locations demonstrating compliance $[\mathrm{X}])$ :

$$
-1=\mathrm{X} \leq 50 \%, 0=50 \%<\mathrm{X} \leq 60 \%, 1=60 \%<\mathrm{X} \leq 70 \%, 2=70 \%<\mathrm{X} \leq 80 \%, 3=\mathrm{X}>80 \% \text {. }
$$

\subsection{Cultural and economic value $[\mathrm{CE}](\mathrm{Ct}$-weight 13\%)}

The cultural and economic value category consists of factors associated with cultural conservation, support of the national economy, and diverse housing typologies. Impacts resulting from lack of cultural conservation, economic 
planning, and housing diversity include: loss of cultural identity, economic stagnancy or decline, land use and contamination, long-term viability of the district (GORD [5]).

Table 5: Cultural and economic value criteria.

\begin{tabular}{|c|c|c|c|}
\hline No. & Criteria & Min. score & Max. score \\
\hline CE.1 & Heritage and cultural identity & -1 & 3 \\
\hline CE.2 & Support of national economy & -1 & 3 \\
\hline CE.3 & Housing diversity & -1 & 3 \\
\hline \multicolumn{2}{|c|}{ Total possible } & -3 & 9 \\
\hline
\end{tabular}

\subsection{4 [CE.3] Housing diversity}

Maximize the diversity of housing typologies within the district to ensure the longterm viability of the development.

Measurement: all projects will develop a variety of housing typologies to promote a range of sustainable living options. The calculator computes the score based on the quantity of different dwelling units for each of the following housing types: single family detached, single family attached, multi-family (GORD [5]).

Score (Simpson diversity index $[\mathrm{X}]$ ):

$$
-1=X<0.6,0=0.6 \leq X<0.65,1=0.65 \leq X<0.7,2=0.7 \leq X<0.75,3=X \geq 0.75 .
$$

\section{Results interpretation and translation}

The initial results can be summarized and interpreted in the following points as enhancing suggestions to the existing GSAS/QSAS tool;

- When performing an assessment for a development on a scale of a city, the process should be performed on a district level, not considering the whole city as one holistic development. Assessing a development in fragmented parts will enable more precise calculation that would make it reliable and valid on the existing site. It will also be more effective to ensure walkability between site amenities, and that the latter ones are sufficient for the whole development.

- The assessment tool should be specific about the scale of the development. Thus the proximity of existing infrastructure is not very important and unrealistic for the new development due to scale difference. The physical condition of existing infrastructure is very important. A development can score 3 points due to the existence of a neighboring development, but at the end both infrastructures are not compatible with each other. In this case the sustainability level of the development is high in terms of quantities, but not on a qualitative basis.

- The tool should consider what type of benefit the development would gain from its proximity to existing ones as the district typology under study has its own existing/planned infrastructure. An analysis of the existing infrastructure 
is required and a detailed investigation is necessary. This analysis and detailed criteria will explain how the new development can actually benefit from existing ones.

- The aim of the bike-ability criteria is to encourage sustainable infrastructure through the development of efficient, user-friendly bicycle pathways. The criterion calculator is not realistic. The calculator assesses this criterion by the length, width, and shading of the bicycle path. The calculator does not mention any relation to the connectivity, suitability or qualities of the pathways.

- $\quad$ For the mixed uses criterion, it should again be calculated district by district and not as a whole project entity. Involving the existing condition of the development, it will achieve higher scores in this criterion if two-fifths $(2 / 5)$ of the districts are extensively mixed, which will increase the use of transportation in the poorly mixed zones. This issue relates back to the first comment where the area of the assessed district should be defined. The study has found that the smaller the district is, the more precise and accurate the assessment is too. Mixed use district should be applied to all development districts to ensure limited car usage and livability of urban public spaces with high quality of life (QoL) standards.

- The public space criterion is a critical one. The calculator depends on the number of users within a grid of $480 \mathrm{~m}$. The criterion aims to enhance social interaction and promote physical and mental well-being of the community by providing accessible and usable outdoor public spaces. Hence, the area of space per capita, and the shading of the public spaces constitute an important issue. Issues such as space perception, quality of urban space, hardscape, landscape, interesting spaces, and the inclusion of social diversity factors are not mentioned or tackled by the calculator. These neglected or omitted issues most likely affect the usability of urban public spaces.

- $\quad$ For the housing diversity criterion; further explanation and specification for the housing typology is needed from the tool to avoid misunderstandings. This is crucial to enable a formulated common language between the client, the GSAS assessor, and the certifier. Furthermore, the client should specify exactly the typology of the housing in his project using the same codes used in the tool; while the assessor must not have the decision of specifying the housing typologies existing in the development. Housing diversity is a very important criterion as it enhances the social healthy living within a community.

- The urban heat island (UHI) effect is not only related to the type of materials, but also to the compactness of the development. The calculator should set standards that specify the adequate distances between existing and planned buildings. This criterion could be enhanced with the addition of another indicator, which is the Sky View Factor (SVF). The SVF is an indicator that quantifies the openness of a site within an urban setting that has significant implications for incoming and outgoing radiation and thus heating and cooling patterns. Reduced SVF increases solar radiation, decreases radiation loss and reduces wind speed. 
- Mitigation strategies for adverse winds as buffer zones should be related to the topography of the land, where the height of the buffer elements effect will vary accordingly. Also, wind buffers should occur regularly within the site especially in open spaces with low rise building and not only on the outer edges.

\section{Conclusion}

This study successfully opens new horizons for sustainability assessment at the urban districts and neighbourhood levels. Furthermore, through the intensive and exhaustive studies carried out in this research, the initial results are encouraging and supportive of developing such innovative emerging tools. Further research should target more and more the macro and meso-scales spatial levels and also targets snapshots as well as time trends in evaluating sustainability levels. The evolution of sustainability tools does not encompass the social aspects and qualitative criteria, and emphasis should be put on this. The study encourages and supports the development of further initiatives, which would help translate and interpret the physical and meta-physical aspects in a unique holistic interactive measurement tool to be applied at diverse locations, scales and temporal elements.

\section{Acknowledgements}

This study was conducted by Ms Maha Sobhey and Ms. Rama Asadi, as part of the term-project of a Master's course on Environmental Impact Assessment. The course was delivered and supervised by Dr. Fodil Fadli (QU) with co-advising from Dr. Elsarrag (GORD).

\section{References}

[1] Abaza, H., Bisset, R., \& Sadler, B., Environmental Impact Assessment and Strategic Environmental Assessment: Towards an Integrated Approach. United Nations Environment Program UNEP: Geneva, pp. 40, (2004).

[2] Abaza, H., Bisset, R., \& Sadler, B., Environmental Impact Assessment and Strategic Environmental Assessment: Towards an Integrated Approach. United Nations Environment Program UNEP: Geneva, pp.7-8, (2004).

[3] Environmental protection Agency EPA, Ireland, http://www.epa.ie /whatwedo/advice/eia/ (accessed 12.1.14)

[4] Gulf Organization for Research and Development GORD, Doha, GSAS/QSAS Technical Guide. http:/gord.qa/uploads/August2012/ Final\%20Draft\%20QSAS\%20Technical\%20Guide.pdf (accessed 13.12.13)

[5] Gulf Organization for Research and Development GORD, Global Sustainability Assessment System-Design Guidelines, GORD: Doha, pp. 11154, (2013).

[6] United Nations Environment Program UNEP, Geneva., http://www.unep.ch/etu/publications/EIA_ovrhds/top02.pdf (accessed 12.1.14) 